\title{
Evaluation of Cellular and Molecular Mechanisms of therapeutic effects of Hibiscus rosa-sinensis and Piper nigrum in Experimental Model of Bronchial Asthma
}

\author{
Pankaj Verma ${ }^{1}$, Kavita Gulati ${ }^{*}$ and Arunabha Ray ${ }^{2}$ \\ ${ }^{1}$ Department of Pharmacology, Vallabhbhai Patel Chest Institute, University of Delhi, Delhi, India \\ ${ }^{2}$ Department of Pharmacology, Hamdard Institute of Medical Sciences and Research (HIMSR), Jamia Hamdard, New Delhi, India \\ *Corresponding Author: Kavita Gulati, Department of Pharmacology, Vallabhbhai Patel Chest Institute, University of Delhi, Delhi, \\ India.
}

Received: January 21, 2022; Published: February 05, 2022

DOI: $10.55162 /$ MCPS.22.028

\begin{abstract}
The present study was performed to experimentally evaluate therapeutic effects of Hibiscus rosa-sinensis and Piper nigrum and their cellular, molecular and bioenhancing effects in ovalbumin (OVA) induced model of bronchial asthma. Wistar rats were sensitized with ovalbumin (OVA) adsorbed on aluminium hydroxide on day 0 followed by OVA challenge on day 14 to experimentally induce bronchial asthma. Hibiscus rosa-sinensis (100 mg/kg and $250 \mathrm{mg} / \mathrm{kg}$ ), Piper nigrum (30 mg/kg and $100 \mathrm{mg} / \mathrm{kg}$ ), combined dose of Hibiscus rosa-sinensis (100 mg/kg) -Piper nigrum (30 mg/kg) and Prednisolone (10 mg/kg) were administered for 14 days and its effects on airway hyper responsiveness in response to spasmogen (methacholine) and airway inflammation were assessed. Enhanced pause ( $p$-enh), a marker of airway hyper responsiveness, was measured in response to inhaled methacholine using whole body plethysmography. Following that rats were anesthetized and blood and bronchoalveolar lavage fluid (BALF) were collected and analyzed for OVA specific IgE and pro-inflammatory cytokine (TNF- $\alpha$ ). Results showed that OVA sensitization and challenge in rats induced increased P-enh levels indicative of enhanced airway hyper responsiveness to methacholine exposure thus validating the experimental model (Disease control). OVA sensitization-challenge treatment resulted in increased IgE and TNF- $\alpha$ in both blood and BAL fluid. Administration of Hibiscus rosa-sinensis (100 mg/kg and $250 \mathrm{mg} / \mathrm{kg}$ ), Piper nigrum (30 $\mathrm{mg} / \mathrm{kg}$ and $100 \mathrm{mg} / \mathrm{kg}$ ) induced dose-dependent attenuations in P-enh levels, IgE as well as TNF- $\alpha$ levels. Combined administration of sub effective doses of Hibiscus rosa-sinensis and Piper nigrum induced significant attenuation in all the parameters in blood and BAL fluid compared to Disease control rats as well as that of herbal drug given alone. The results showed that Hibiscus rosa-sinensis and Piper nigrum reduced both (a) airway hyper-responsiveness to the bronchoconstrictor; and (b) the biomarkers of airway inflammation and validates the observed therapeutic benefits of this Indian traditional medicinal plant for bronchial asthma
\end{abstract}

Keywords: Bronchial asthma; Airway Hyper responsiveness; Ovalbumin; Methacholine; Enhanced Pause

\section{Introduction}

Bronchial asthma is a complex chronic inflammatory disease of the airways characterized by airway inflammation, reversible airflow obstruction and bronchial hyper responsiveness and regulated by several cellular and humoral factors (Kim et al., 2011; Holgate et al., 2008)[15,13]. It involves recruitment and activation of many inflammatory and structural cells such as mast cells, macrophages, eosinophils, T-lymphocytes, neutrophils, epithelial and smooth muscle cells. Some of these cells are capable of synthesizing and releasing inflammatory mediators that are central to the pathophysiology of the disease. For example, T-lymphocytes (particularly Th2

Citation: Kavita Gulati., et al. "Evaluation of Cellular and Molecular Mechanisms of therapeutic effects of Hibiscus rosa-sinensis and Piper nigrum in Experimental Model of Bronchial Asthma”. Medicon Pharmaceutical Sciences 2.2 (2022): 02-13. 
Evaluation of Cellular and Molecular Mechanisms of therapeutic effects of Hibiscus rosa-sinensis and Piper nigrum in Experimental Model of Bronchial Asthma

type) and mast cells produce various cytokines (IL-4, IL-5, TNF- $\alpha$ etc.), which are crucial humoral players in the process. Th2 cytokines including IL-4, IL-5 and IL-13 produced by activated CD4+ T-cells, play a central role in the pathogenesis of asthma by controlling the key processes of immunoglobulin E (IgE) production, growth, differentiation and activation of mast cells and eosinophils (Lemanske et al., 2010)[17]. Pharmacotherapy of bronchial asthma consists of anti-inflammatory agents (corticosteroids) and bronchodilators $(\beta$-2-agonists). However, significant incidence of adverse effects related to these drugs has been a major area of concern. In addition, the increasing incidence of refractoriness to conventional forms of therapy has further complicated the problem. As a result, search for newer and more viable alternatives from herbal sources has been insurge for the control of bronchial asthma. It has been observed that many herbal products are being used by folklore which seems to be effective for several diseases. Therefore, the literature was surveyed and Hibiscus rosa-sinensis and Piper nigrum were selected on the basis of their traditional use in Indian traditional system of medicine. These products need to be scientifically validated by modern methodology for integration in the main stream of the medical health care system.

Hibiscus rosa-sinensis is a bushy, evergreen shrub or small tree with glossy leaves, red flowers in summer and autumn. Moreover, Hibiscus rosa-sinensis have been traditionally used for bronchial asthma in most parts of North Eastern India (Khan et al., 2010)[14]. Hibiscus rosa-sinensis has been shown to contain vital essential nutrients such as calcium, magnesium, zinc, potassium which can help in the management of bronchial asthma (Sahito et al., 2013)[26]. Piper nigrum (Dried pepper) has been extensively used for its flavoring properties as well as traditional medicine. Aqeuous extract of fruits of Sapindus mukorossi and Piper nigrum have been reported to show anti-asthmatic properties by inhibiting acetylcholine induced bronchoconstriction of isolated goat trachea (Parganiha et al., 2012)[24]. Another study with P. nigrum as an ingredient in a polyherbal compound has reported significant invitro and in-vivo anti-histaminic and bronchodilator activity in albino rats (Amutha et al., 2015)[2]. Bhatt et al., [4] used Piper nigrum as an ingredient in a polyherbal formulation, Zeal Herbal Granules and reported anti-asthmatic effect through mast-cell stabilization properties (Bhatt et al., 2013)[4].

The present study was conducted to validate the anti-asthma potentials of Hibiscus rosa-sinensis and Piper nigrum and evaluate the cellular and molecular mechanism in an experimental model of bronchial asthma in rats.

\section{Materials and Methods \\ Animals}

Wistar rats of either sex, weighing 180-220 g were used for the study. They were housed in standard laboratory cages and kept in environmentally controlled room $\left(25 \pm 2^{\circ} \mathrm{C}, 12\right.$ hours light and dark cycle). Animals were acclimatized for one week before treatment. They were fed with standard laboratory food pellets and water ad libitum. The study protocols were approved by Institutional Animal Ethics Committee (VPCI/IAEC/2017/15), following the guidelines of CPCSEA (Committee for the purpose of control and supervision of experiments on animals), which complies with international guidelines of Indian National Science Academy (INSA), New Delhi.

\section{Drugs and Chemicals}

The test drugs Hibiscus rosa-sinensis and Piper nigrum were provided by Amsar Pvt. Ltd., India. Ovalbumin, Methacholine and Prednisolone were procured from Sigma Aldrich-USA. All other routine chemicals were procured from SRL, New Delhi. Cytokine assay kits such as Ovalbumin specific IgE and TNF- $\alpha$ were procured from Weldon Biotech, New Delhi.

\section{OVA-induced model of airway inflammation}

All the animals in each group were immunized with intraperitoneal injection of Ovalbumin $(10 \mathrm{mg} / \mathrm{rat}) \mathrm{emulsified}$ with $10 \mu \mathrm{g}$ of aluminium hydroxide on day 0 . Herbal agent, Hibiscus rosa-sinensis and Piper nigrum and prednisolone (10 mg/kg) were given daily on separate groups for 14 days. The animals were challenged with ovalbumin (1 mg) in $0.5 \mathrm{ml}$ of isotonic saline on $14^{\text {th }}$ day (Kwasnieski et al., 1998)[16].

Citation: Kavita Gulati., et al. "Evaluation of Cellular and Molecular Mechanisms of therapeutic effects of Hibiscus rosa-sinensis and Piper nigrum in Experimental Model of Bronchial Asthma”. Medicon Pharmaceutical Sciences 2.2 (2022): 02-13. 
Evaluation of Cellular and Molecular Mechanisms of therapeutic effects of Hibiscus rosa-sinensis and Piper nigrum in Experimental Model of Bronchial Asthma

\section{Experimental Protocol}

Rats were divided into 8 groups ( $\mathrm{n}=5$ rats per group) viz., (i) Normal control (NC): rats were sensitized with ovalbumin on day 0 and treated orally with distilled water for 14 days(vehicle); (ii) Disease control (DC): rats were sensitized (day 0) and challenged with ovalbumin (day 14) and treated orally with distilled water for 14 days (vehicle); (iii)Positive Control (PC): rats were sensitized (day 0) and treated orally with prednisolone at the dose of $10 \mathrm{mg} / \mathrm{kg}$ from day 1 to 14, followed by challenge with ovalbumin (day 14), (iv) and (v) Hibiscus rosa-sinensis (100) and (250) groups: rats were sensitized and treated orally with Hibiscus rosa-sinensis at the dose of 100 $\mathrm{mg} / \mathrm{kg}$, or $250 \mathrm{mg} / \mathrm{kg}$ from day 1 to 14, followed by challenge with ovalbumin; (vi) and (vii) Piper nigrum (30) and (100) groups: rats were immunized/sensitized and treated orally with Piper nigrum at the dose of $30 \mathrm{mg} / \mathrm{kg}$, or $100 \mathrm{mg} / \mathrm{kg}$ from day 1 to 14, followed by challenge with ovalbumin; (viii) Hibiscus-Piper: rats were immunized and treated orally with combined dose of Hibiscus rosa-sinensis at the dose of $100 \mathrm{mg} / \mathrm{kg}$ and Piper nigrum at the dose of $30 \mathrm{mg} / \mathrm{kg}$, from day 1 to 14 , followed by challenge with ovalbumin in all experimental groups. The dosage of Piper nigrum and Hibiscus rosa-sinensis were selected on the basis of range of doses mentioned in the literature used for experimental studies (Bui et al., 2020; Mani et al., 2016; Mishra et al., 2012; Verma et al., 2016) [8,19,21,31].

After $24 \mathrm{~h}$ of ovalbumin sensitization, Enhanced Pause (P-enh), a marker of airway hyperresponsiveness was measured in response to inhaled methacholine using whole body plethysmography (Hammelmann et al., 1997)[12]. P-enh is an index of bronchial hyperresponsiveness and airway resistance in experimental animals evaluated using whole body plethysmography. Briefly, rats were placed in a whole-body chamber and basal readings were obtained and averaged for a 3 min period. Subsequently, increasing doses of methacholine $(2.5 \mathrm{mg} / \mathrm{ml}, 10 \mathrm{mg} / \mathrm{ml}$ and $20 \mathrm{mg} / \mathrm{mL})$, was aerosolized for 3 minutes, and readings were taken and averaged for $3 \mathrm{~min}$ after each nebulization. Following whole body plethysmography, rats were sacrificed to collect blood and BAL samples (Vos et al., 2007)[32].

After evaluating P-enh using whole body plethysmography, the animals were used to evaluate markers of airway inflammation and immunity in both blood and BAL fluid. The animals were anaesthetized with ketamine ( $24 \mathrm{mg} / \mathrm{kg}$, i.p.), blood and BAL fluid samples were collected. Blood samples (4-6 ml) were collected through cardiac puncture and were centrifuged at $4 \stackrel{\circ}{\circ} \mathrm{C}(3000 \mathrm{rpm})$ for $10 \mathrm{~min}$ to separate the serum and stored at $-80^{\circ}$ C.BAL fluid was collected by lavaging the lung through a tracheal cannulation with $0.9 \%$ sodium chloride solution and centrifuged at $1500 \mathrm{rpm}$ at $4^{\circ} \mathrm{C}$ for $10 \mathrm{~min}$ and supernatant was recovered and stored at $-80^{\circ} \mathrm{C}$ for assay of various biochemical markers (Abdureyim et al., 2011)[1].

\section{Assay for TNF- $\alpha$}

TNF- $\alpha$ in Blood and BAL fluid samples was analyzed by using commercially available enzyme linked immonosorbent assay (ELISA) kits as per manufacturer's instructions. TNF- $\alpha$ level was measured using sandwich ELISA method. Antigen and biotin-conjugated polyclonal antibody preparation specific for TNF- $\alpha$ was added to microtiter plate pre-coated with polyclonal antibody specific to TNF- $\alpha$ and incubated for specified periods. Then, streptavidin horse-radish peroxidase and TMB substrate were added to produce a colored reaction product. The-enzyme-substrate reaction was stopped by adding sulphuric acid. The absorbance of the colored product was read at a wavelength of $450 \mathrm{~nm}$ using ELISA plate reader and values were expressed in $\mathrm{pg} / \mathrm{ml}$.

\section{Assay for OVA-specific IgE}

OVA-specific IgE (OVAsIgE) or allergan specific IgE is used to assess the role of allergen in aggravating IgE levels in experimental animals of bronchial asthma. OVA-specific IgE (OVAsIgE) in serum and BAL fluid were analysed by using commercially available ELISA test kits as per manufacturer's instructions. Briefly, the microtiter plate was pre-coated with an antibody specific to OVA-specific IgE (OVAsIgE). Standard, Test samples and HRP-labeled conjugate specific for OVA-specific IgE (OVAsIgE) were simultaneously incubated for specified periods. Then, Chomogen A and B were added to produce a coloured reaction. The absorbance was read at a wavelength of $450 \mathrm{~nm}$ using ELISA plate reader and results were expressed as $\mathrm{ng} / \mathrm{ml}$.

Citation: Kavita Gulati., et al. "Evaluation of Cellular and Molecular Mechanisms of therapeutic effects of Hibiscus rosa-sinensis and Piper nigrum in Experimental Model of Bronchial Asthma”. Medicon Pharmaceutical Sciences 2.2 (2022): 02-13. 
Evaluation of Cellular and Molecular Mechanisms of therapeutic effects of Hibiscus rosa-sinensis and Piper nigrum in Experimental Model of Bronchial Asthma

\section{Statistical analysis}

Statistical analysis was performed using appropriate parameteric and non-parametric tests. A p value of $\leq 0.5$ was taken as level of significance in all the statistical tests perform.

\section{Results}

Effects of Hibiscus rosa-sinensis and Piper nigrum on spasmogen induced bronchial hyperresponsiveness

Enhanced Pause (P-enh), a marker of bronchial hyper-responsiveness was measured using whole body plethysmography at different dose levels of methacholine $(2.5 \mathrm{mg} / \mathrm{ml}, 5 \mathrm{mg} / \mathrm{ml}, 10 \mathrm{mg} / \mathrm{ml}$ and $20 \mathrm{mg} / \mathrm{ml})$. Sensitization of rats with OVA followed by challenge treatment resulted in increased degree of enhanced pause $(P$-enh), when measured in response to exposure to different doses of methacholine as compared to normal control rats. Treatment with both the doses of Hibiscus rosa-sinensis (100 mg/kg and $250 \mathrm{mg} / \mathrm{kg}$ ) for 14 days resulted in attenuation of $P$-enh values at different levels of methacholine exposure. However, significant reduction in $P$-enh levels were observed with higher dose of Hibiscus rosa-sinensis (250 mg/kg) as compared to that of Disease control rats as shown in Figure 1(a). Similarly, Piper nigrum (30 mg/kg and $100 \mathrm{mg} / \mathrm{kg}$ ) was administered for 14 days and its effect on enhanced pause (P-enh) was evaluated using whole body plethysmography. Administration of Piper nigrum (30 mg/kg and $100 \mathrm{mg} / \mathrm{kg}$ ) resulted in dose-dependent reduction of P-enh level in OVA sensitized and challenged rats with significant reduction of P-enh level with higher dose of Piper nigrum i.e. $100 \mathrm{mg} / \mathrm{kg}$ as shown in Figure 1(b). Subeffective doses of Hibiscus rosa-sinensis (100 mg/kg) and Piper nigrum (30 mg/kg) when combined resulted in significant reduction of $P$-enh levels as compared to that of disease control rats. Thus, lower doses of both Hibiscus rosa-sinensis and Piper nigrum when administered alone, did not show any marked effect but when administered in combination resulted in marked reduction of $P$-enh levels as compared to that of disease control rats as shown in Figure 1(c). In addition, prednisolone treated group showed significant attenuation in P-enh levels in response to all doses of methacholine (basal-20 mg/ml) as compared to that of Disease control rats as shown in Figure $1(\mathrm{p}<0.05)$

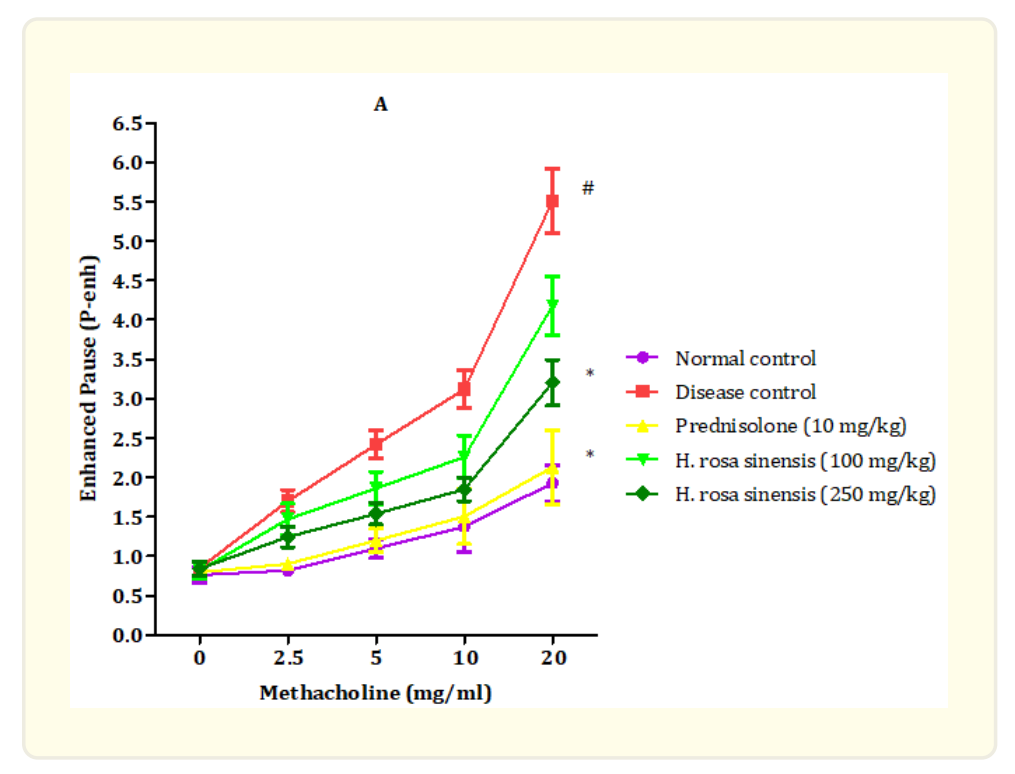

Citation: Kavita Gulati., et al. "Evaluation of Cellular and Molecular Mechanisms of therapeutic effects of Hibiscus rosa-sinensis and Piper nigrum in Experimental Model of Bronchial Asthma”. Medicon Pharmaceutical Sciences 2.2 (2022): 02-13. 
Evaluation of Cellular and Molecular Mechanisms of therapeutic effects of Hibiscus rosa-sinensis and Piper nigrum in Experimental Model of Bronchial Asthma

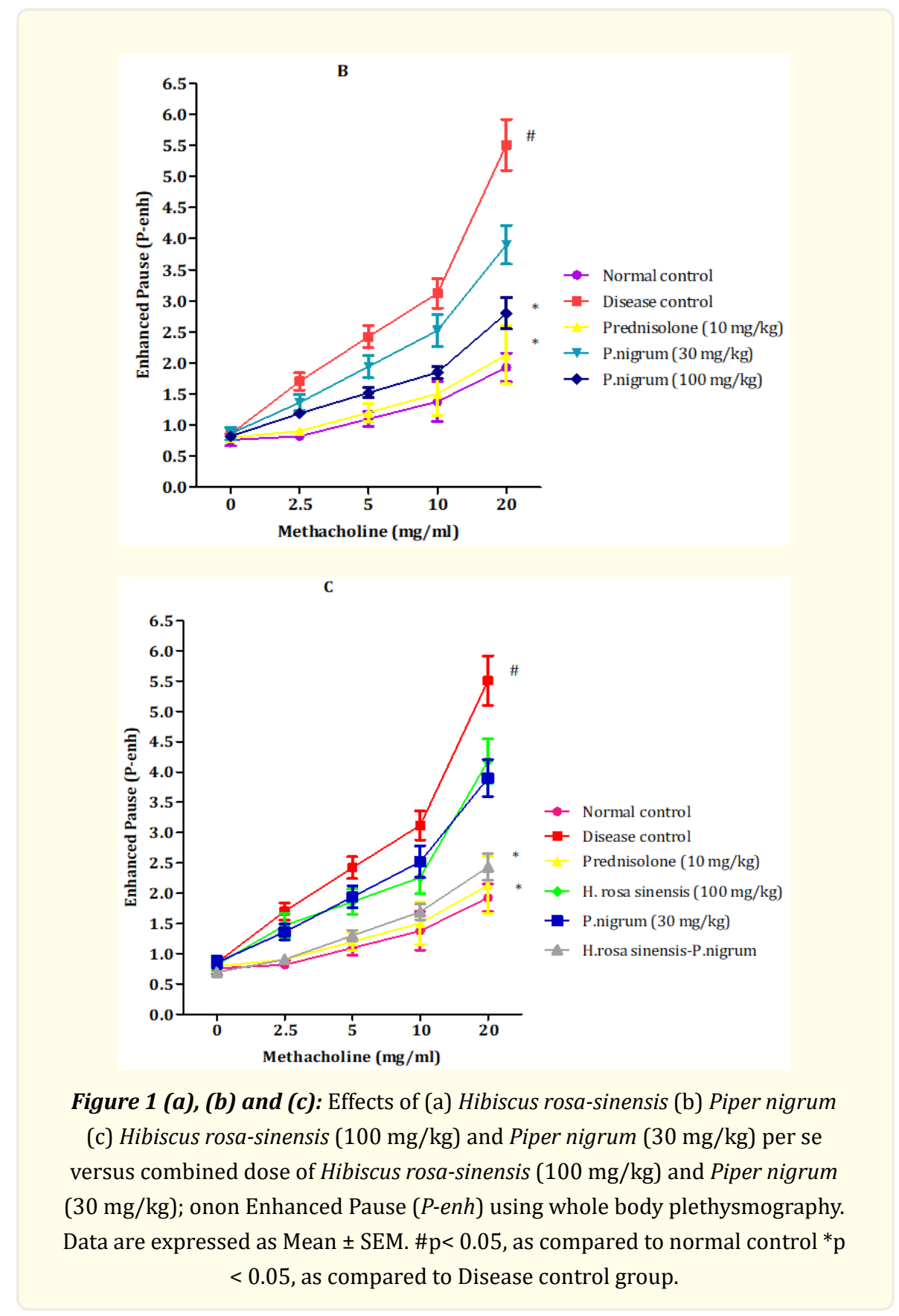

\section{Estimation of Ovalbumin Specific IgE (OVAsIgE) in Blood and BAL fluid}

OVA-specific IgE or allergan specific IgE helps to assess the role of allergen in aggravating IgE levels in experimental animals of bronchial asthma. Assay for OVA-specific IgE showed OVA sensitized and challenged rats resulted in significant increase in IgE levels in blood and BAL fluid in Disease control rats compared to that of normal control rats $(\mathrm{p}<0.05)$. Administration of Hibiscus rosa-sinensis $(100 \mathrm{mg} / \mathrm{kg}$ and $250 \mathrm{mg} / \mathrm{kg}$ ) showed dose-dependent attenuation/reduction of IgE levels in blood and BAL fluid as compared to IgE 
Evaluation of Cellular and Molecular Mechanisms of therapeutic effects of Hibiscus rosa-sinensis and Piper nigrum in Experimental Model of Bronchial Asthma

level of disease control rats. However, significant attenuation in IgE levels was seen only with higher dose of Hibiscus rosa-sinensis (250 $\mathrm{mg} / \mathrm{kg}$ ) in both blood and BAL fluid as compared to that of Disease control rats as shown in figure $2(\mathrm{a})(\mathrm{p}<0.05)$. Similarly, pretreatment with Piper nigrum (30 mg/kg and $100 \mathrm{mg} / \mathrm{kg}$ ) induced dose-dependent attenuation of IgE levels by $30 \%$ (30 mg/kg) and 40\% $(100 \mathrm{mg} / \mathrm{kg}$ ) in blood and by $26 \%$ (30 mg/kg) and 31\% (100 mg/kg) in BAL fluid as compared to that of disease control rats. However, Piper nigrum at $100 \mathrm{mg} / \mathrm{kg}$ only achieved significant attenuation of IgE levels in blood and BAL fluid as compared to that of Disease control rats as shown in figure 2(b) ( $\mathrm{p}<0.05)$. Combination of lower doses of Hibiscus rosa-sinensis (100 mg/kg) and Piper nigrum (30 $\mathrm{mg} / \mathrm{kg}$ ) administered for 14 days resulted in attenuation of IgE levels by $48 \%$ in blood and $32 \%$ in BAL fluid as compared to that of disease control rats. Although, both doses of Hibiscus rosa-sinensis and Piper nigrum when administered alone, did not show any significant effect when administered in combination resulted in significant reduction of IgE levels in blood and BAL fluid versus disease control rats as shown in Figure 2(c). The results were comparable with prednisolone treated group which showed significant attenuation in IgE levels by $43 \%$ in blood and $36 \%$ in BAL fluid as compared to that of Disease control rats as shown in Figure 2 ( $\mathrm{p}<0.05$ ).

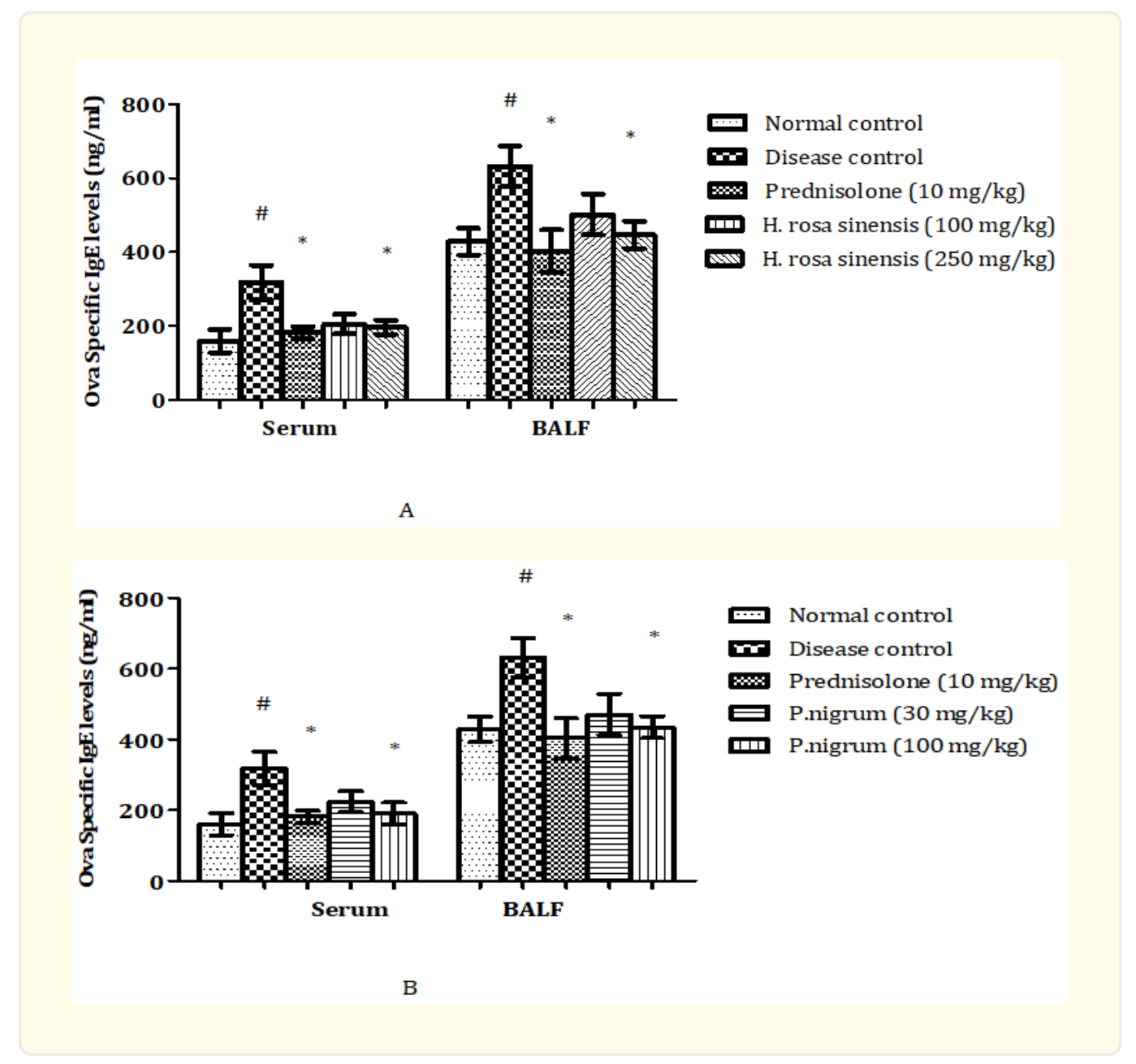

Citation: Kavita Gulati., et al. "Evaluation of Cellular and Molecular Mechanisms of therapeutic effects of Hibiscus rosa-sinensis and Piper nigrum in Experimental Model of Bronchial Asthma". Medicon Pharmaceutical Sciences 2.2 (2022): 02-13. 


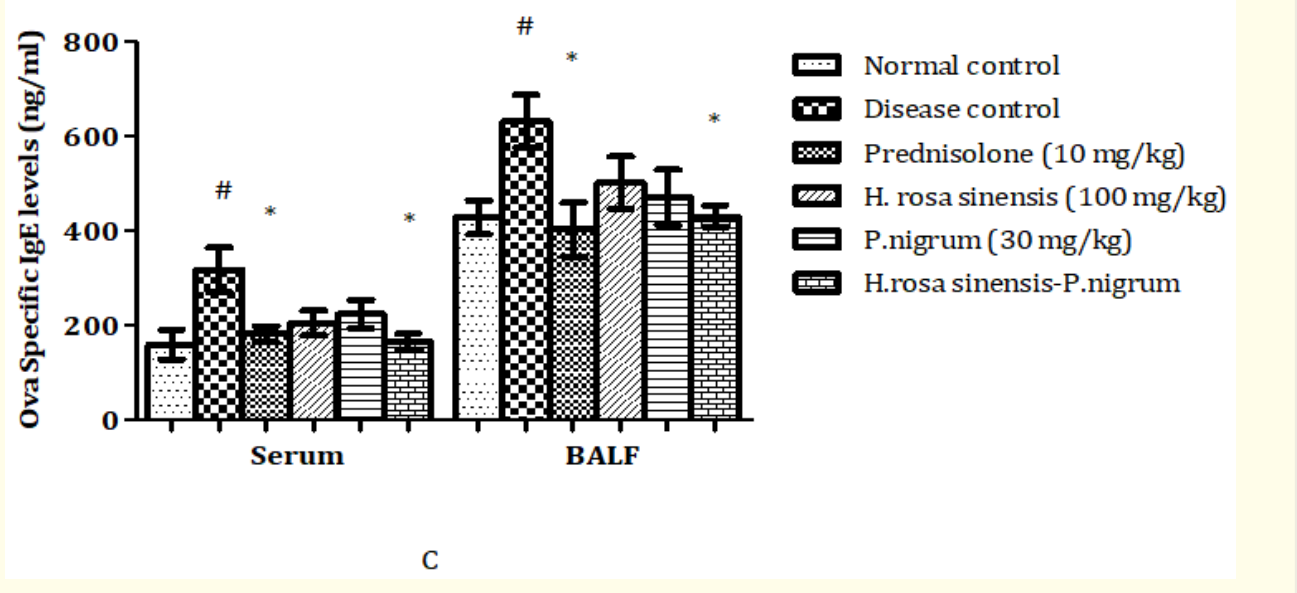

Figure 2: Effects of (a) Hibiscus rosa-sinensis (b) Piper nigrum (c) Hibiscus rosa-sinensis (100 mg/ $\mathrm{kg}$ ) and Piper nigrum (30 mg/kg) per se versus combined dose of Hibiscus rosa-sinensis (100 mg/ $\mathrm{kg}$ ) and Piper nigrum (30 mg/kg); on Ovalbumin specific IgE levels in blood and BAL fluid of OVA immunized and challenged rats. Data are expressed as Mean $\pm \mathrm{SEM}$. $\# \mathrm{p}<0.05$, as compared to normal control ${ }^{*} \mathrm{p}<0.05$, as compared to disease control group.

Assay of TNF- $\alpha$

Assay of TNF- $\alpha$ showed significantly high level of TNF- $\alpha$ in both blood and BAL fluid in OVA sensitized and challenged rats as compared to that in normal rats $(\mathrm{p}<0.05)$. However, prior treatment with Hibiscus rosa-sinensis $(100 \mathrm{mg} / \mathrm{kg}$, and $250 \mathrm{mg} / \mathrm{kg}) \mathrm{showed}$ dose-dependent attenuation in TNF- $\alpha$ level by $9 \%$ and $24 \%$ in blood and $15 \%$ and $23 \%$ in BAL fluid respectively as compared to Disease control rats. Hibiscus rosa-sinensis $(250 \mathrm{mg} / \mathrm{kg}$ ) showed significant attenuation of IgE levels in blood and BAL fluid as compared to that of Disease control rats as shown in figure 3(a) ( $\mathrm{p}<0.05)$. Similarly, pre-treatment with Piper nigrum induced attenuations in TNF- $\alpha$ level by $14 \%$ and $33 \%$ in blood and $20 \%$ and $29 \%$ in BAL fluid as compared to Disease control rats. However, significant attenuation in TNF- $\alpha$ level was seen with higher dose of Piper nigrum $(100 \mathrm{mg} / \mathrm{kg}$ ) only in both blood and BAL fluid as compared to that of Disease control rats as shown in figure 3(b). Similar to earlier results with IgE, Combination of sub effective doses of Hibiscus rosa-sinensis (100 $\mathrm{mg} / \mathrm{kg}$ ) and Piper nigrum (30 mg/kg) showed significant reduction in TNF- $\alpha$ levels by $36 \%$ and $31 \%$ in both blood and BAL fluid as compared to that of disease control rats as shown in figure $3(\mathrm{c})(\mathrm{p}<0.05)$. Prednisolone-treated rats also showed significant reduction in TNF- $\alpha$ levels by $35 \%$ and $33 \%$ in blood and BAL fluid as compared to disease control rats $(\mathrm{p}<0.05)$. 


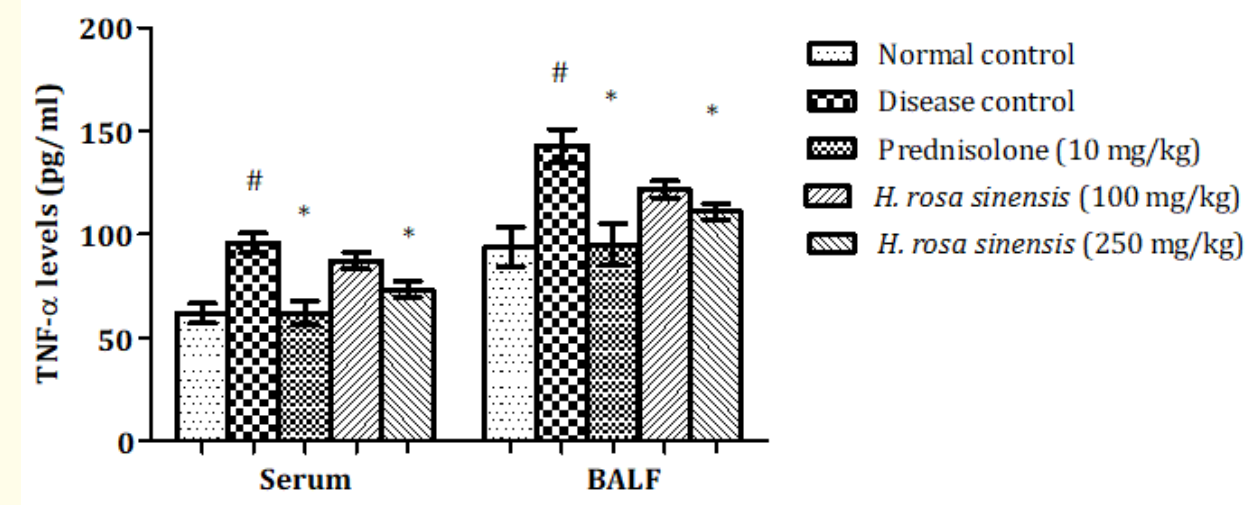

A
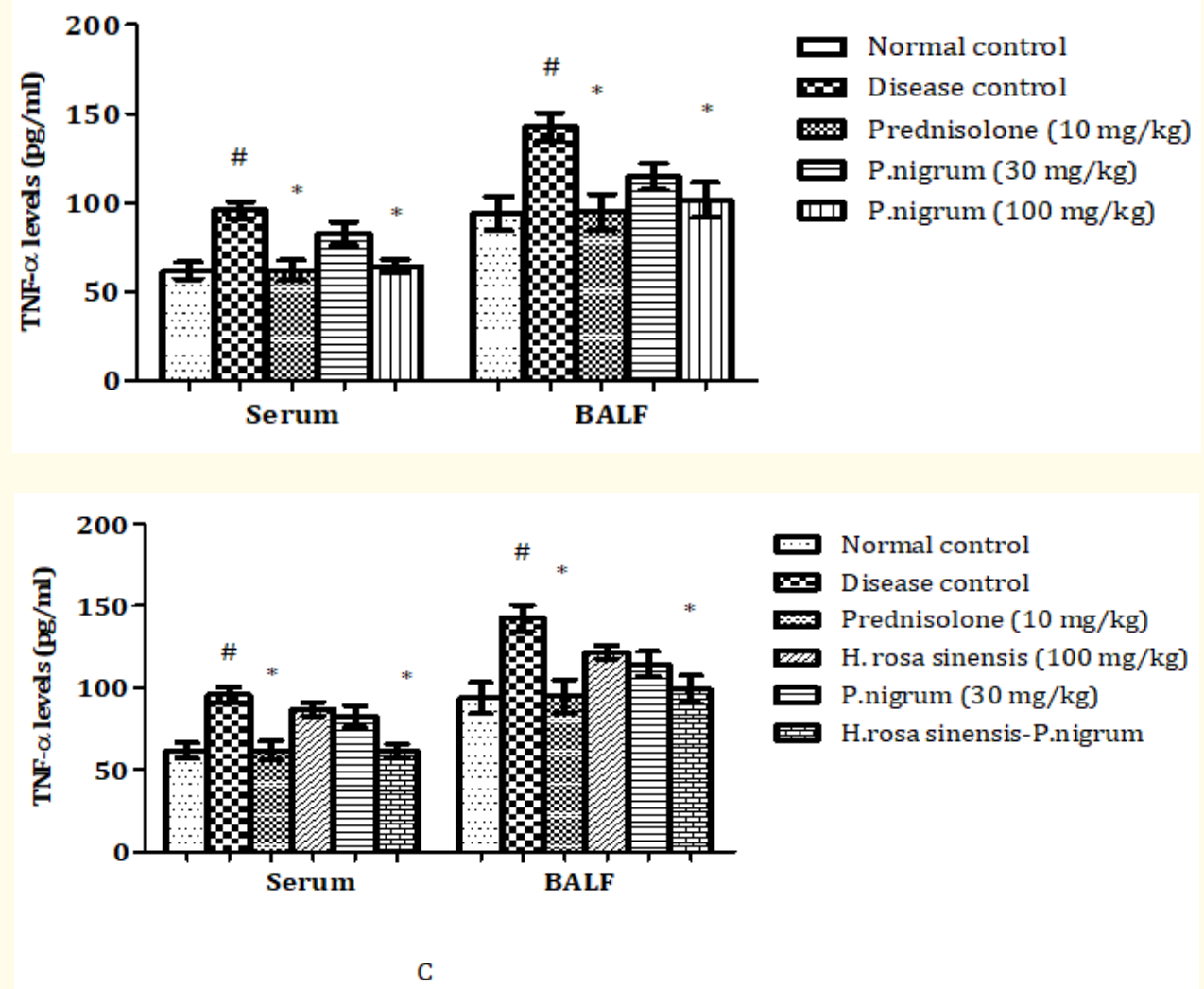

Figure 3: Effects of (a) Hibiscus rosa-sinensis (b) Piper nigrum (c) Hibiscus rosa-sinensis (100 mg/ $\mathrm{kg}$ ) and Piper nigrum (30 mg/kg) per se versus combined dose of Hibiscus rosa-sinensis (100 mg/ $\mathrm{kg}$ ) and Piper nigrum (30 mg/kg); on TNF- $\alpha$ levels in blood and BAL fluid of OVA immunized and challenged rats. Data are expressed as Mean \pm SEM. \#p< 0.05 , as compared to normal control * $\mathrm{p}<$ 0.05 , as compared to disease control group. 
Evaluation of Cellular and Molecular Mechanisms of therapeutic effects of Hibiscus rosa-sinensis and Piper nigrum in Experimental Model of Bronchial Asthma

\section{Discussion}

Bronchial asthma is a disease of the airways mainly characterized by airway obstruction, airway inflammation as well as airway hyperresponsiveness mediated through cellular and humoral events. Exposure to various allergens results in a cascade of event resulting in a Th-2 type mediated immune response which releases various inflammatory mediators such as chemokines and cytokines from mast cells, inflammatory cells (eosinophils and neutrophils) which trigger symptoms into an individual. Pharmacotherapy of bronchial asthma mainly requires long term treatment with controllers (corticosteroids) and relievers (beta agonists) but these are associated with various side effects and refractoriness has also been reported (Barnes et al., 1993; Boulet et al., 1998)[3,6]; which in turn raised concern towards finding alternatives with better efficacy and safety. Therefore, search for newer and more viable alternatives from herbal sources has been growing for the control of bronchial asthma. Various studies have also reported role of herbal drugs such as Albizzia lebbeck, Solanum xanthocarpum, Adianutm venustum, Lychnis coronaria, UNIM-352, Piper longum etc. in improving asthma symptoms as well as markers of bronchial asthma in experimental rats (Chaudhary et al., 2016; Rai et al., 2015; Gulati et al., 2021; Verma et al., 2021) [9,25,11,30]. Therefore, the literature was surveyed and Hibiscus rosa-sinensis and Piper nigrum were selected on the basis of their traditional use in Indian traditional system of medicine to evaluate their therapeutic effects on cellular and molecular markers of bronchial asthma using modern methodology. Moreover, the bioenhancing effect of combined dose of Hibiscus rosa-sinensis and Piper nigrum was also evaluated using standard experimental models of bronchial asthma. This study was performed to evaluate various anti-inflammatory and immunomodulatory markers to determine the underlying molecular and cellular mechanism involved in their therapeutic effects.

Hibiscus rosa-sinensis is traditionally used for bronchial asthma in many parts of North Eastern India (Khan et al., 2010)[14]. Hibiscus rosa-sinensis has been shown to contain vital essential nutrients such as calcium, magnesium, zinc, potassium which can help in the management of bronchial asthma (Sahito et al., 2013)[26]. Piper nigrum (Dried pepper) has been extensively used for its flavoring properties as well as traditional medicine. Aqeuous extract of fruits of Sapindus mukorossi and Piper nigrum have been shown to have anti-asthmatic properties as evident by inhibiting acetylcholine induced bronchoconstriction of isolated goat trachea (Parganiha et al., 2012)[24]. Another study of Piper nigrum as one of the ingredient in a polyherbal compound reported significant in vitro and in-vivo anti-histaminic and bronchodilator activity in albino rats (Amutha et al., 2015)[2].

In present study, Enhanced Pause (P-enh), a marker of airway hyperresponsiveness was measured in response to aerosolized spasmogen i.e. methacholine $(0,2.5 \mathrm{mg} / \mathrm{ml}, 5 \mathrm{mg} / \mathrm{ml}, 10 \mathrm{mg} / \mathrm{ml}$ and $20 \mathrm{mg} / \mathrm{ml})$ using whole body plethysmography and averaged for 3 minutes. Enhanced pause is positively correlated to airway AHR and airway resistance (Finkelmann et al., 2008; Mckinley et al., 2004) $[10,20]$. OVA sensitized and challenged mice are reported to have higher enhanced pause value which are positively correlated with airway neutrophilia and airway epithelial injury (Nui et al., 2019)[23]. In present study, OVA sensitized and challenged rats showed significant increase in enhanced pause in response to methacholine as compared to that of normal control rats. Administration of Hibiscus rosa-sinensis and Piper nigrum for 14 days resulted in significant reduction of P-enh levels as compared to that of Disease control rats. Further, significant reduction in P-enh levels were seen with administration of combined subeffective dose of Hibiscus rosa-sinensis (100 mg/kg) and Piper nigrum (30 mg/kg). These results were comparable with prednisolone, the positive control.

Inhibition of inflammatory response and symptomptomatic relief remains one of the main stay for the anti-asthmatic drugs. Immunization of rats followed by antigen challenge treatment were performed to experimentally simulate bronchial asthma and induce release of cytokines as well as inflammatory response, marked by mobilization of inflammatory cells (eosinophils and neutrophils) and release of cytokines such as TNF- $\alpha$, IL-4, IL-5,IL-13, IFN- $\Upsilon$ etc. IgE is a reaginic antibody which plays vital role in airway inflammation and other allied allergic reactions. Higher levels of IgE are reported in adults and children with asthma and are associated with greater asthma severity, airway hyper responsiveness and lower baseline lung function (Borisch et al., 2005; Naqvi et al., 2007)[5,22]. TNF- $\alpha$ has been known to act as chemo attractant for neutrophils and eosinophils, also involved in the activation of T-cells and increases epithelial expression of adhesion molecules (Lukacs et al., 1995; Scheurich et al., 1987) [18,27]. It has been reported that levels of TNF- $\alpha$ mRNA and protein were elevated in airways of asthmatic patients (Bradding et al., 1994; Ying et al., 1991)[7,29]. Moreover, there has

Citation: Kavita Gulati., et al. "Evaluation of Cellular and Molecular Mechanisms of therapeutic effects of Hibiscus rosa-sinensis and Piper nigrum in Experimental Model of Bronchial Asthma". Medicon Pharmaceutical Sciences 2.2 (2022): 02-13. 
Evaluation of Cellular and Molecular Mechanisms of therapeutic effects of Hibiscus rosa-sinensis and Piper nigrum in Experimental Model of Bronchial Asthma

been evidence for airway hyper responsiveness and airway neutrophilia in normal healthy subjects resulted due to administration of inhaled recombinant TNF- $\alpha$ (Thomas et al., 2002; Thomas et al., 1995)[28,29]. OVA Sensitization followed by challenge treatment increased the IgE and TNF- $\alpha$ levels in blood and BAL fluid of experimental animals. Interestingly, pretreatment with herbal agents, Hibiscus rosa-sinensis $(100 \mathrm{mg} / \mathrm{kg}$ and $250 \mathrm{mg} / \mathrm{kg}$ ) reduced the levels of IgE in blood and BAL fluid as compared to that in disease control rats. Further, combined administration of sub effective dose of Hibiscus rosa sinensis (100 mg/kg) and Piper nigrum (30 mg/ $\mathrm{kg}$ ) also showed significant reduction of OVA specific IgE and TNF- $\alpha$ levels in blood and BAL fluid as compared to that of disease control rats, thus suggesting bioenhancing effects of Piper nigrum. These results were comparable with standard drug, Prednisolone, which also showed reduction inhyperresponsiveness to spasmogen and markers of inflammation and immunomodulation. Similarly, our previous studies have also shown that OVA sensitization and challenge treatment resulted in elevated/increased levels of IgE, TNF- $\alpha$, IL-4, inflammatory cells (eosinophil and neutrophils) in blood and BAL fluid. Further, treatment with different doses of herbal agents such as UNIM-352, Albizzia lebbeck, Solanum xanthocarpum, Adiantum venustum etc resulted in attenuation of these cytokine levels in both blood and BAL fluid (Chaudhary et al., 2016; Rai et al., 2015; Gulati et al., 2021; Verma et al., 2021) [9, 25, 11, 30].

\section{Conclusion}

Taken together, the study shown that treatment with Hibiscus rosa-sinensis and Piper nigrum reduced levels of inflammatory markers such as IgE and TNF- $\alpha$ in both blood and BALF, thus validating the anti-inflammatory and immunomodulatory effects of the herbal drugs. Moreover, whole body plethysmography showed that P-enh values were also lowered following Hibiscus rosa-sinensis and Piper nigrum treatment in experimental models of bronchial asthma, thus validating its therapeutic efficacy which is reportedly used as a folklore medicine in North-eastern India.

\section{Acknowledgement}

The authors gratefully acknowledge Indian Council of Medical Research (ICMR), India for funding the senior research fellowship to Mr. Pankaj Verma and MRU-DHR-VPCI for providing the ELISA kits (IgE and TNF- $\alpha$ ). The authors are also thankful to AMSAR Pvt. Ltd. for providing standardized plant extracts.

\section{Conflict of Interest}

Authors have no personal or financial conflicts of interest in relation to the publication of this manuscript.

\section{References}

1. Abdureyim S., et al. "Anti-inflammatory, immunomodulatory, and heme oxygenase-1 inhibitory activities of ravannapas, a formulation of uighur traditional medicine, in a rat model of allergic asthma”. Evidence Based Complementary and Alternative Medicine (2011): 1-13.

2. Amutha A., et al. "Investigation into the studies on antihistaminic and bronchodilator activity of polyherbal compound (Siringiyathi Chooranam) in albino rats". Journal of Chemical and Pharmaceutical Research 7.9 (2015): 777-781.

3. Barnes PJ., et al. "Efficacy and safety of inhaled corticosteroids in asthma”. American Review of Respiratory Disease 148.4 (1993): S01-S26.

4. Bhatt S., et al. "Evaluation of acute toxicity study and antiasthmatic activity of Zeal Herbal granules". International Research Journal of Pharmacy 4.5 (2013): 213-215.

5. Borish L., et al. "Total serum IgE levels in a large cohort of patients with severe or difficult-to-treat asthma". Annals of Allergy and Asthma Immunology 95.3 (2005): 247-53.

6. Boulet LP. "Perception of the role and potential side effects of inhaled corticosteroids among asthmatic patients". Chest Journal 113.3 (1998): 587-592.

7. Bradding P., et al. "Interleukin-4, -5 , and -6 and tumor necrosis factor-alpha in normal and asthmatic airways: evidence for the

Citation: Kavita Gulati., et al. "Evaluation of Cellular and Molecular Mechanisms of therapeutic effects of Hibiscus rosa-sinensis and Piper nigrum in Experimental Model of Bronchial Asthma”. Medicon Pharmaceutical Sciences 2.2 (2022): 02-13. 
Evaluation of Cellular and Molecular Mechanisms of therapeutic effects of Hibiscus rosa-sinensis and Piper nigrum in Experimental Model of Bronchial Asthma

human mast cell as a source of these cytokines". American Journal of Respiratory Cellular and Molecular Biology 10 (1994): 471-80.

8. Bui TT., et al. "Piper Nigrum extract improves OVA-induced nasal epithelial barrier dysfunction via activating Nrf2/HO-1 signaling". Cellular Immunology 351 (2020): 104035.

9. Chaudhary S., et al. "Evaluation of Anti-Inflammatory and Immunomodulatory Effects of Aqueous Extract of Solanum Xanthocarpum in Experimental Models of Bronchial Asthma” EC Pharmacology and Toxicology 2.6 (2016): 241-250.

10. Finkelman FD. "Use of unrestrained, single chamber barometric plethysmography to evaluate sensitivity to cholinergic stimulation in mouse models of allergic airway disease". Journal of Allergy and Clinical Immunology 121.2 (2008): 334-335.

11. Gulati K., et al. "Evaluation of anti-inflammatory and immunomodulatory potential of a traditional herbal drug in experimental model of bronchial asthma”. EC Pharmacology and Toxicology 9.4 (2011): 130-138.

12. Hamelmann E., et al. "Noninvasive measurement of airway responsiveness in allergic mice using barometric plethysmography". American Journal of Respiratory and Critical Care Medicine 156.3 (1997): 766-775.

13. Holgate ST., et al. "Epithelial mesenchymal interactions in the pathogenesis of asthma". Journal of Allergy and Clinical Immunology 52.2 (2008): 45-52.

14. Khan MH., et al."Herbal remedies of asthma in Thoubal district of Manipur in North East India" Indian Journal of Natural Product Research 1.1 (2010): 80-84.

15. Kim H., et al. "Asthma”. Allergy Asthma and Clinical Immunology 7.1 (2011): 1-9.

16. Kwasniewski FH., et al. "Impairment in connective tissue mast cells degranulation in spontaneously hypertensive rats: stimulus dependent resistance”. British Journal of Pharmacology 124 (1998): 772-778.

17. Lemanske RF., et al. "Asthma: Clinical expression and molecular mechanisms" Journal of Allergy and Clinical Immunology 125 (2010): S95-102.

18. Lukacs NW., et al. "TNF- $\alpha$ lpha mediates recruitment of neutrophils and eosinophils during airway inflammation". Journal of Immunology 154.10 (1995): 5411-427.

19. Mani A., et al. "Role of phyto-stabilised silver nanoparticles in suppressing adjuvant induced arthritis in rats". International Immunopharmacology.41 (2016): 17-23.

20. McKinley L., et al. "Reproducibility of a novel model of murine asthma-like pulmonary inflammation". Clinical and Experimental Immunology 136 (2004): 224-231.

21. Mishra N., et al. "Immunomodulation by Hibiscus rosa-sinensis: effect on the humoral and cellular immune response of Mus musculus". Pakistan Journal of Biological Sciences 15.6 (2012): 277-83.

22. Naqvi M., et al. "Association between IgE levels and asthma severity among African American, Mexican, and Puerto Rican patients with asthma". Journal of Allergy and Clinical Immunology 120.1 (2007): 137-43.

23. Nui C., et al. "Enhanced pause correlates with airway neutrophils and airway-epithelial injury in asthmatic mice treated with dexamethasone" Journal of Asthma 56.1 (2019): 11-20.

24. Parganiha R., et al. "In vitro anti-asthmatic activity of fruit extracts of Sapindus mukorossi and Piper nigrum" International Journal of Herbal Drug Research 1.3 (2012): 12-16.

25. Rai N., et al. "Cellular and Molecular mechanism of action of polyherbal preparation UNIM-352 in experimental models of Bronchial Asthma" Indian Journal of Experimental Biology 53.10 (2015): 625-631.

26. Sahito SB., et al."Determination and Evaluation of Mineral Constituents of Medicinal Plants used for the Treatment of Asthma and other Ailments by Atomic Absorption Spectrophotometry" Pakistan Journal of Analytical and Environmental Chemistry 14.1 (2013): 61-67.

27. Scheurich P., et al. "Immunoregulatory activity of recombinant human tumor necrosis factor (TNF)-alpha: induction of TNF receptors on human T cells and TNF- $\alpha$ lpha-mediated enhancement of T cell responses". Journal of Immunology 138 (1987):178690.

28. Thomas PS., et al. "Tumor necrosis factor-alpha increases airway responsiveness and sputum neutrophilia in normal human

Citation: Kavita Gulati., et al. "Evaluation of Cellular and Molecular Mechanisms of therapeutic effects of Hibiscus rosa-sinensis and Piper nigrum in Experimental Model of Bronchial Asthma”. Medicon Pharmaceutical Sciences 2.2 (2022): 02-13. 
subjects". American Journal of Respiratory Cellular and Molecular Biology 152.1 (1995): 76-80.

29. Thomas PS, et al. "Effects of inhaled tumour necrosis factor alpha in subjects with mild asthma". Thorax 57.9 (2002): 774-8.

30. Verma P., et al. "Evaluation of cellular and molecular mechanism of anti-asthmatic effects of a traditional herbal drug in rats". Asian Journal of Pharmaceutical Research and Development 9.5 (2021): 29-34.

31. Verma P., et al. "Anti-inflammatory effect of hydroalcoholic extract of hibiscus rosa on acute and chronic inflammation". International Journal of Pharmacology and Toxicology 4.2 (2016): 123-26.

32. Vos AP., et al. "Dietary supplementation with specific oligosaccharide mixtures decreases parameters of allergic asthma in mice". International Immunopharmacology 7.12 (2007): 1582-1587.

33. Ying S., et al. "TNF alpha mRNA expression in allergic inflammation”. Clinical and Experimental Allergy 21.6 (1991): $745-50$.

Volume 2 Issue 2 February 2022

(C) All rights are reserved by Kavita Gulati., et al. 